
\title{
28 Research Suare \\ Nonlinear Low Rank Representation by Estimating Optimal Transformations for Image Clustering
}

\section{Haoran Chen}

Zhengzhou University of Light Industry

\section{Xu Chen}

Zhengzhou University of Light Industry

Hongwei Tao ( $\square$ tthhww_811@163.com )

Zhengzhou University of Light Industry

\section{Zuhe Li}

Zhengzhou University of Light Industry

\section{Hanming Guo}

Zhengzhou University of Light Industry

\section{Yong Cui}

Zhengzhou University of Light Industry

\section{Research Article}

Keywords: Subspace Clustering, Low Rank Representation, Alternative Conditional Expectation, Nonlinear Transformation

Posted Date: January 20th, 2022

DOI: https://doi.org/10.21203/rs.3.rs-1205778/v1

License: (c) (i) This work is licensed under a Creative Commons Attribution 4.0 International License.

Read Full License 


\title{
Nonlinear low rank representation by estimating optimal transformations for image clustering
}

\author{
Haoran $\mathrm{Chen}^{1}$, Xu Chen ${ }^{1}$, Hongwei Tao ${ }^{1 * \dagger}$, Zuhe \\ $\mathrm{Li}^{1{ }^{*} \dagger}$, Hanming Guo ${ }^{1^{*} \dagger}$ and Yong Cui ${ }^{1 * \dagger}$ \\ ${ }^{1 *}$ College of Computer and Communication Engineering, \\ Zhengzhou University of Light Industry, Science Road, \\ Zhengzhou, 450000, Henan, CHINA.
}

*Corresponding author(s). E-mail(s): tthhww_811@163.com; zuheli@126.com; 2724543503@qq.com; goodaliens@126.com;

Contributing authors: chenhaoran@zzuli.edu.cn;

ccxx_1208@163.com;

$\dagger$ These authors contributed equally to this work.

\begin{abstract}
Low rank representation is a very effective method, which is often used in unsupervised learning and semi-supervised classification. Due to the presence of noise and nonlinearity structure in real data, low rank representation fails to obtain the global information of the data well, so it can not be able to get enough discrimination information. For this motivation, this paper proposes a novel nonlinear low rank representation by estimating optimal transformations (EOTLRR) which can obtain a better global structure of the dataset by increasing the correlation among samples that may belong to the same category. The proposed method establishes an objective function that maximizes the largest q-ky Fan norm of the covariance matrix of data samples through estimating optimal transformations. Alternative Conditional Expectation method is employed to maximize the conditional expectation of samples, which can increase the correlation among the samples belonged to the same category. Experimental results on five real datasets and a synthetic dataset show that our method has superior performance compared with other latest methods.
\end{abstract}


Keywords: Subspace Clustering, Low Rank Representation, Alternative Conditional Expectation, Nonlinear Transformation

Acknowledgments. The work is supported by the Natural Science Foundation of China under Grant No. 61906175, and the Key Technologies R\&D Program of Henan Province under Grant No. 212102210409.

\section{Introduction}

In recent years, subspace clustering has attracted extensive attention from academia and researchers, and it has many applications in various fields such as machine learning (Chen, Mao, Zhang, \& Yi, 2018; Zhu \& Peng, 2020) and computer vision (Du, Ma, Li, \& Wang, 2020). For instance, an image of a cat is composed of hundreds of thousands of pixels, which are located in a low-dimensional subspace of the surrounding space. The purpose of subspace clustering is to segment high-dimensional data into corresponding low-dimensional subspaces, so as to understand the intrinsic structure of high-dimensional data. After decades of development, the current subspace clustering methods used in computer vision can be divided into algebra-based methods (Vidal, Ma, \& Sastry, 2005), deep learning-based methods (Peng et al., 2018, 2020), statistical methods (Michael \& Christopher, 1999; A.Y. Yang, Rao, \& Yi, 2006) and spectral clustering methods (Lauer \& Schnorr, 2009; J. Zhang, Li, Jing, Liu, \& Su, 2018; Zhong \& Pun, 2020).

Spectral clustering methods have been widely used because of their excellent performance such as facial recognition and image segmentation (Du et al., 2020; Feng, Meng, \& Wang, 2020), can be achieved by two steps: 1) obtain the similarity matrix which use the linear combination of each sample; 2) use the spectral clustering algorithm to obtain the clustering results such as K-means (Shamir \& Tishby, 2010), Normalized Cut (N-cut) (Shi \& J.Malik, 2000 ) and so on. These methods mainly consider the use of Euclidean distance and Gaussian kernel function to measure the similarity among samples. Ideally, this similarity matrix should be a block diagonal matrix, i.e., the similarity among classes is zero. In other words, how to get a better similarity matrix is a challenging problem. In fact, numerous clustering methods based on spectral clustering have been developed over the past few decades. The most classic methods based on spectral clustering are low rank representation (LRR) (G. Liu et al., 2010) and sparse subspace clustering (SSC) (Elhamifar \& Vidal, 2013). SSC tries to obtain the sparse similarity matrix. However, its disadvantage mainly is that it cannot guarantee the sparsity of each data sample when pursuing the overall sparseness of the similarity matrix.

Low rank representation, as an effective method to obtain the low dimensional structure of high dimensional data, has attracted extensive attention in multi-classification. The purpose of LRR is to find the lowest rank of the similarity matrix, which is used for various feature learning tasks and has achieved 
impressive clustering results (Chen et al., 2018; G. Liu et al., 2013; Xue et al., 2019; Yu, Tan, Zhang, Tao, \& Rui, 2019; Yu, Tao, Wang, \& Rui, 2015). LRR chooses the data itself as a dictionary to get the similarity matrix. However, this method may be relatively ill-behaved when the data is insufficient, noisy, and / or non-linear structure. To solve the problem of noise and the insufficient data, (G. Liu \& Yan, 2011) proposed a latent LRR (lat LRR) method. The method utilized observed data and hidden unobserved data as a dictionary to learn features. Although lat LRR improves the clustering performance, it has many solutions. In order to get the best solution, robust latent LRR (H. Zhang, Lin, Zhang, \& Gao, 2014) chooses the most sparse solution as the final result. Nevertheless, when the number of samples is insufficient, it may lead to poor results. To overcome this shortcoming, Liu et al. got inspiration from matrix decomposition and proposed a fixed rank representation (R. Liu, Lin, la Torre, \& Su, 2012) method, which introduced a sparse regular term to ensure the robustness of the clustering algorithm. When there are noisy data, it can obtain a closed-form of multi subspace structure.

In addition, various variant methods based on LRR have a poor performance when dealing with data of nonlinear spatial structure. (Wang, Saligrama, \& A., 2011) proposed using kernel technique to transform the original dataset into linear spatial structure by nonlinear mapping and got a satisfactory clustering performance. Xiao et al. proposed robust kernel low rank representation (Xiao, Tan, Xu, \& Dong, 2016) to deal with data structures in nonlinear space. It used kernel technology to map data from original space to multiple linear subspaces. However, different kernel functions may be suitable for different datasets. Besides, there are some scholars suggest that the method of graph regularization is used to deal with the data of nonlinear structure. For instance, (Yin, Gao, \& Lin, 2016) proposed Laplacian regularized LRR (lap LRR) by combining the Laplacian graph with the LRR model. (Li, Cui, \& Dong, 2017) proposed the graph regular non-negative low rank matrix factorization model (GNLMF) to improve the robustness of data in the manifold structure.

When the data noise-free and a linear space structure, the traditional LRR method can effectively obtain the global structure of the data. However, the noise and nonlinearity of datasets always exist in practical application. To reduce the influence of nonlinearity and noise in the data on the LRR model, we find an efficient nonlinear transformation method which can be competent to do the work proposed in (Breiman \& Friedman, 1985a), which is called by Estimating Optimal Transformations (EOT). EOT is aim to fit the nonlinear transformation function by maximizing the correlation between labels and samples which have been transformed nonlinearly into linearly space. As a special case of estimating optimal transformations, Maximally Correlated Principle Component Analysis (MCPCA) (Feizi \& Tse, 2017) considers the maximum correlation among samples after nonlinear transformation, which can enhance the correlation among samples in the same class. 
Based on the aforementioned analysis, this paper proposes a nonlinear low rank representation by estimating optimal transformations (EOTLRR) method for subspace clustering. The main contributions of this paper are:

1. We propose to use the EOT method to eliminate the influence of nonlinear structure of datasets by maximizing the correlation between nonlinear transferred data.

2. We propose the EOTLRR method which learns effectively the low rank affinity matrix of the datasets and has better performance and robustness compared with other latest methods on several real datasets and a synthetic dataset.

The remaining part of this paper is organized as follows: In Section 2, we give a brief introduction about the estimating optimal transformations fuction and solve it by using alternating conditional expectation. In Section 3, we introduce the proposed EOTLRR algorithm for subspace clustering. Then, the experimental results and analysis are given in Section 4. Finally, the conclusions are summarized in Section 5.

\section{Related works}

EOT method is introduced in this section, which can be implemented the Alternating Conditional Expectation (ACE) (Breiman \& Friedman, 1985b). ACE considers maximation amongs samples with conditional expectation, which can increase the samples correlation of the same category. Besides, the ACE algorithm as a parameter-free automatic conversion method, it produces the maximum correlations of between samples and labels.

The EOT method solves the general problem establishing a linear hypothesis of regression analysis and better describes the relationship between the independent and dependent variables and can best describe the presence of a nonlinear relationship. Let $\mathbf{y}$ be a response variable and $\mathbf{x}_{1}, \mathbf{x}_{2}, \ldots, \mathbf{x}_{p}$ be predictors. Let $\theta(\mathbf{y}), \phi_{1}\left(\mathbf{x}_{1}\right), \ldots, \phi_{p}\left(\mathbf{x}_{p}\right)$ denote the zero mean transformation function for response and predictors variable. The fraction of variance not explained is

$$
e^{2}\left(\theta, \phi_{1}, \ldots, \phi_{p}\right)=\frac{E\left\{\left[\theta(\mathbf{y})-\sum_{i=1}^{p} \phi_{i}\left(\mathbf{x}_{i}\right)\right]\right\}}{E \theta^{2}(\mathbf{y})}
$$

These functions are real values. When the original variable is categorical, the use of $\theta$ or $\phi_{i}$ assign a real value score to each categorical value. The EOT method are only based on the data sample $\left\{\left(Y_{k}, X_{k 1}, \cdots, X_{k p}\right), 1 \leq k \leq N\right\}$ in which contains minimum assumptions the data distribution regarding the form of the optimal transformation. The optimal transformations are defined to be the equator that minimize

$$
e^{2}\left(\theta, \phi_{1}, \ldots, \phi_{p}\right)=\frac{E\left\{\left[\theta\left(X_{p+1}\right)-\sum_{j=1}^{p} \phi_{j}\left(X_{j}\right)\right]^{2}\right\}}{E \theta^{2}\left(X_{p+1}\right)}
$$


these data consist of $N+p$ successive observations $\mathbf{x}=\left[\mathbf{x}_{1}, \mathbf{x}_{2}, \ldots, \mathbf{x}_{N+p}\right]$. This exists in the form of standard data by defining $Y_{k}=x_{k+p}, X_{k}=$ $\left[X_{k+p-1}, \ldots, X_{k}\right]$. the optimal solution is found by iterations. Suppose the distribution of the variable $\mathbf{y}, \mathbf{x}_{1}, \mathbf{x}_{2}, \ldots, \mathbf{x}_{p}$ is known. Without losing generality, let the expectation of all functions be zero, and $E \theta^{2}(\mathbf{y})=1$. For better illustration, the unexplained error fraction in bivariate case can be expressed as

$$
e^{2}(\theta, \phi)=E[(\mathbf{y})-\phi(\mathbf{x})]^{2}
$$

In view of finding the minimum value of the problem(3) with respect to $\theta(\mathbf{y})$ under a given function $\phi(\mathbf{x})$. This solution can be expressed as

$$
\theta_{1}(\mathbf{y})=E[\phi(\mathbf{x}) \mid \mathbf{y}] / \| E[\phi(\mathbf{x}) \mid \mathbf{y} \|]
$$

where $\|\cdot\|=\left[E(\cdot)^{2}\right]^{1 / 2}$. Next, considered finding the minimum value of the problem(4) with respect to $\mathbf{x}$ under a given function $\mathbf{y}$. This solution can be expressed as

$$
\phi_{1}(\mathbf{x})=E[\theta(\mathbf{y}) \mid \mathbf{x}]
$$

Problem(4) and (5) form the basis of (ACE) concerning iterative optimization process. In the basic ACE algorithm, the function is minimized alternately while keeping the other function fixed, thereby reducing problem(2) in each iteration. Then discuss the case of multiple predictors $\mathbf{x}_{1}, \mathbf{x}_{2}, \ldots, \mathbf{x}_{p}$ and conduct direct analogy with the basic ACE algorithm. The minimization function can be expressed by the following,

$$
e^{2}\left(\theta, \phi_{1}, \ldots, \phi_{p}\right)=E\left[\theta(\mathbf{y})-\sum_{j=1}^{p} \phi_{j}\left(\mathbf{x}_{j}\right)\right]^{2}
$$

where keeping $E \theta^{2}=1, E \theta=E \phi_{1}, \ldots,=E \phi_{p}=0$ by minimizing a series of single functions involving bivariate conditional expectation. As for given functions $\phi_{1}\left(\mathbf{x}_{1}\right), \ldots, \phi_{p}\left(\mathbf{x}_{p}\right)$, minimize the value of problem(6) with respect to $\phi(\mathbf{y})$

$$
\theta_{1}(\mathbf{y})=E\left[\sum_{i=1}^{p} \phi_{i}\left(\mathbf{x}_{i}\right) \mid \mathbf{y}\right] / \| E\left[\sum_{i=1}^{p} \phi_{i}\left(\mathbf{x}_{i}\right) \mid \mathbf{y} \|\right]
$$

Then for a given $\theta(\mathbf{y})$ and a given set $\phi_{1}, \ldots, \phi_{k}, \ldots, \phi_{p}$ a single function $\phi_{k}\left(\mathbf{x}_{k}\right)$ Problem(6) minimization. The solution is

$$
\theta_{k, 1}\left(\mathbf{x}_{k}\right)=E\left[\theta(\mathbf{y})-E \sum_{i \neq k} \phi_{i}\left(\mathbf{x}_{i}\right) \mid \mathbf{x}_{k}\right]
$$

For the function $\phi_{k}, k=1, \ldots, p$, each iteration of the inner loop For both of problem (6) is minimized, other functions are fixed. The iteration of outer loop will continue until the predictor variables with one complete pass fails to decrease $e^{2}$. The specific details of the ACE are in Algorithm1. 


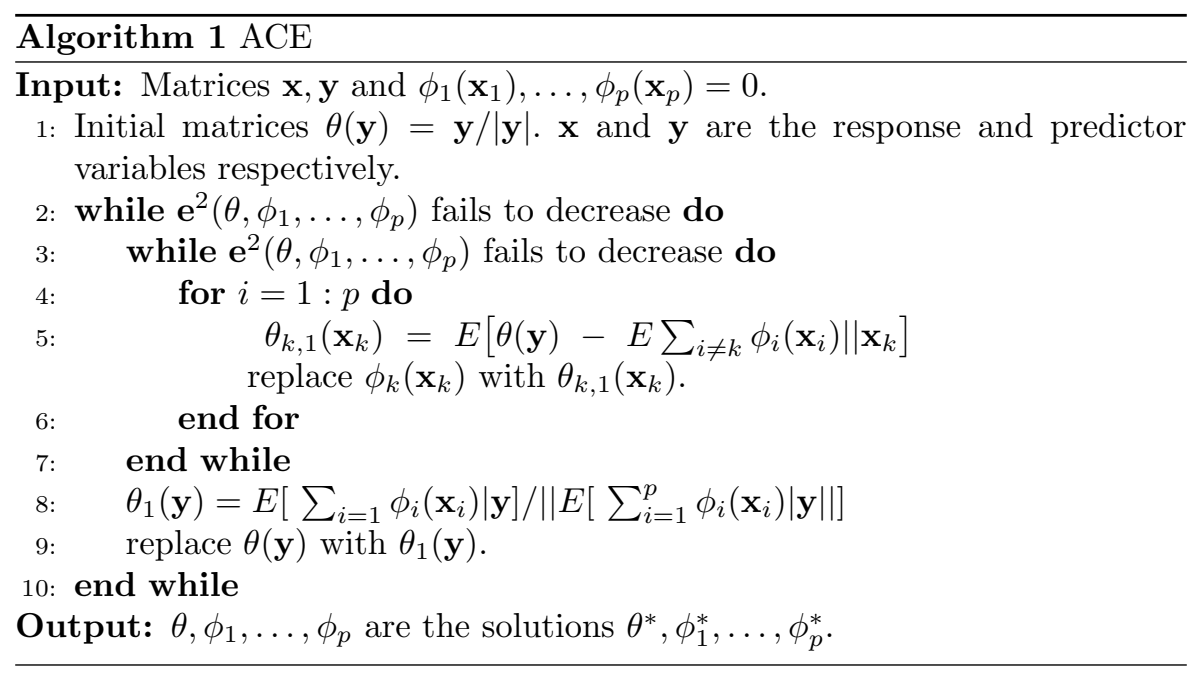

\section{Nonlinear Low Rank Representation by Estimating Optimal Transformations}

In this section, a detailed description of the EOTLRR method for subspace clustering is given. Our purpose is to gain a similar matrix by using LRR technology efficiently utilizing the transformed data. Let a set of data $\mathbf{X}=$ $\left[\mathbf{x}_{1}, \mathbf{x}_{2}, \ldots, \mathbf{x}_{n}\right] \in \mathbb{R}^{m \times n}(i=1,2, \ldots, n)$ be the original data, each column of which is a sample ( $\mathbf{x}_{i}$ denotes the $i$-th sample), $m$ and $n$ are the number of features and sample, respectively.

\subsection{Estimating Optimal Transformations}

Estimating optimal transformations aims at finding possibly nonlinear transformations of the data, which can minimize the mean squared error between the transformed data and its low rank approximation by $p$ orthonormal vectors $v_{1}, \ldots, v_{p}$. The mean square error between the low rank approximations obtained by this vector is as follows

$$
\begin{aligned}
& \min _{\left\{v_{i}\right\}_{i=1}^{p},\left\{\varphi_{i}\right\}_{i=1}^{p}} \frac{1}{n} \sum_{k=1}^{n}\left\|\varphi\left(\mathbf{x}_{k}\right)-\hat{\varphi}\left(\mathbf{x}_{k}\right)\right\|^{2} \\
& \text { s.t. } \quad \hat{\varphi}\left(\mathbf{x}_{k}\right)=\sum_{i=1}^{p}\left(\mathbf{v}_{i}^{T} \varphi\left(\mathbf{x}_{k}\right)\right) \mathbf{v}_{i}, \quad 1 \leq k \leq n \\
& \varphi\left(\mathbf{x}_{k}\right)=\left(\varphi_{1}\left(\mathbf{X}_{k, 1}\right), \ldots, \varphi_{q}\left(\mathbf{X}_{k, q}\right)\right), \\
& \mathbf{v}_{i}^{T} \mathbf{v}_{j}=0, \quad 1 \leq i \neq j \leq p, \\
& \mathbf{v}_{i}^{T} \mathbf{v}_{i}=1, \quad 1 \leq i \leq p,
\end{aligned}
$$


Nonlinear low rank representation by estimating optimal transformations for image clusteri

$$
\begin{aligned}
& \frac{1}{n} \sum_{k=1}^{n} \varphi_{i}\left(\mathbf{X}_{k, i}\right)=0, \quad 1 \leq i \leq q, \\
& \frac{1}{n} \sum_{k=1}^{n} \varphi_{i}\left(\mathbf{X}_{k, i}\right)^{2}=1, \quad 1 \leq i \leq q .
\end{aligned}
$$

Where the additional constraint $\frac{1}{n} \sum_{k=1}^{n} \varphi_{i}\left(\mathbf{X}_{k, i}\right)=0$ is analogous to the normal process, in which columns of the data matrix are transformed to be zero means with experience. The additional constraint $\frac{1}{n} \sum_{k=1}^{n} \varphi_{i}\left(\mathbf{X}_{k, i}\right)^{2}=1$ enable columns of the transformed matrix to have the same norms. Let $\mathbf{F}=\left[F_{1}, \ldots, F_{q}\right]$ be a discrete variable and $\mathbf{G}=\left[G_{1}, \ldots, G_{q}\right]$ be $q$ finite discrete random variables. $\mathbf{P}_{F_{1}, \ldots, F_{q}}$ and $\mathbf{P}_{H_{1}, \ldots, H_{q}}$ are expressed by represent the joint distribution of $\mathbf{F}$ and the joint probability distribution of $\mathbf{G}$, respectively. $\mathbf{P}_{H_{1}, \ldots, H_{q}}$ is equal to the observed empirical distribution samples. i.e.,

$$
\mathbf{P} r\left(H_{1}=j_{1}, \ldots, H_{p}=j_{q}\right)=\frac{1}{n} \sum_{k=1}^{n} \mathbf{1}\left\{H_{k, 1}=j_{1}, \ldots, H_{k, p}=j_{q}\right\}
$$

Besides, the estimating optimal transformations method can be calculated by using the empirical pair joint distribution, but the calculation cost of this method is very expensive. Therefore, estimating optimal transformations optimization can be written as

$$
\begin{aligned}
& \max _{\left\{\varphi_{i}\right\}_{i=1}^{q},\left\{\mathbf{v}_{i}\right\}_{i=1}^{p}} \sum_{r=1}^{p} \sum_{i=1}^{q} \sum_{i^{\prime}=1}^{q} v_{r, i} v_{r, i^{\prime}}\left(\frac{1}{n} \sum_{s=1}^{n} \varphi_{i}\left(\mathbf{X}_{s, i}\right) \varphi_{i^{\prime}}\left(\mathbf{X}_{s, i^{\prime}}\right)\right) \\
& \frac{1}{n} \sum_{s=1}^{n} \varphi_{i}\left(\mathbf{X}_{s, i}\right)=0, \quad 1 \leq i \leq q \\
& \frac{1}{n} \sum_{s=1}^{n} \varphi_{i}\left(\mathbf{X}_{s, i}\right)^{2}=1, \quad 1 \leq i \leq q \\
& \mathbf{v}_{r}^{T} \mathbf{v}_{s}=0, \quad 1 \leq r \neq s \leq p \\
& \mathbf{v}_{r}^{T} \mathbf{v}_{r}=1, \quad 1 \leq r \leq p
\end{aligned}
$$

The specific details of the Solving estimating optimal transformations are in Algorithm2.

\subsection{EOTLRR}

The affinity matrix obtained from LRR is a linear representation of each data point under the global low-rank constraint. Consequently, LRR is suitable for obtaining the global structure of the linear structure data. The LRR can be expressed as

$$
\min _{\mathbf{Z}, \mathbf{E}}\|\mathbf{Z}\|_{l}+\lambda\|\mathbf{E}\|_{l}, \quad \text { s.t. } \mathbf{X}=\mathbf{A} \mathbf{Z}+\mathbf{E}
$$




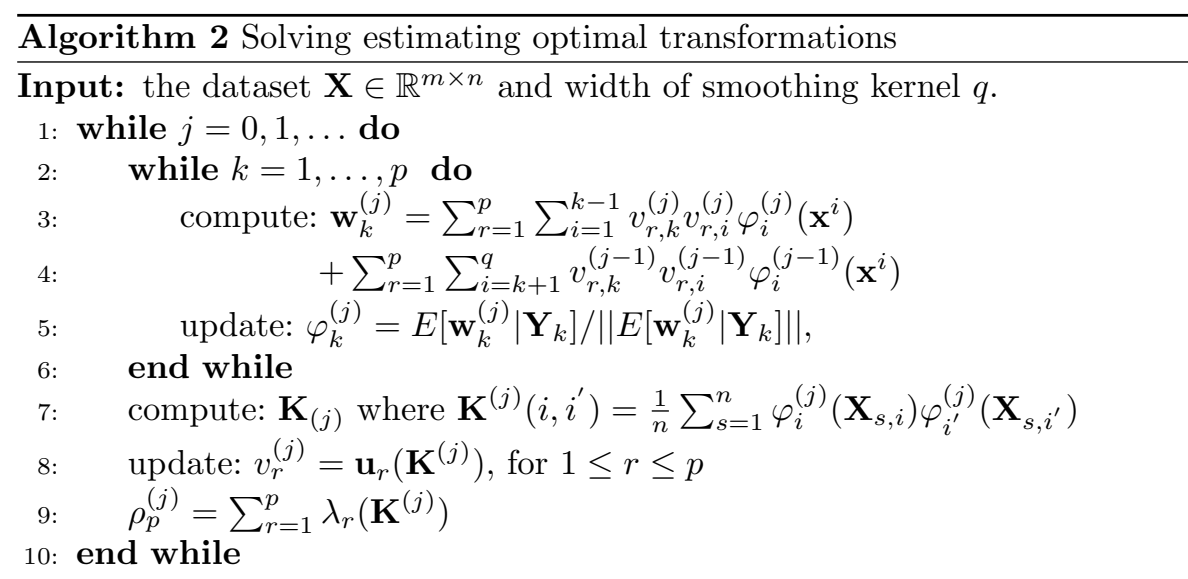

Output: $\mathbf{X}^{*}$ is the result of the estimating optimal transformations of the dataset $\mathbf{X}$

Where $\|\bullet\|_{l}$ is the regularization strategy nuclear and $\mathbf{A}$ is the dictionary, generally $\mathbf{A}=\mathbf{X} . \lambda>0$ is parameter used to balance low rank terms and $\mathbf{E}$ is the error matrix for noise. $\mathbf{Z}=\left[\mathbf{z}_{1}, \ldots, \mathbf{z}_{n}\right]$ is the representation matrix of the data. EOT can be used to increase the similarity between samples belonging to the same category, so a "good" affinity matrix can be obtained. The estimating optimal transformations can map the same dimensional data original nonlinear into linear, fully considering the global structure of the observation sample. $\varphi(\cdot)$ is defined as the estimating optimal transformations function, which can be solved by ACE in Section 2. Hence the representation matrix of nonlinear transformed data can be expressed as

$$
\varphi\left(\mathbf{X}_{i, j}\right)=\varphi\left(\mathbf{X}_{i, j}\right) \mathbf{Z}_{i, j}
$$

where $\mathbf{X}_{i, j}$ represents the pixel of the image, and $\mathbf{Z}_{i, j}$ is the correlation coefficient between the $i$-th and $j$-th samples. $\mathbf{Z}$ is the similarity matrix of LRR gained through problem (12). $\varphi(\mathbf{X})$ represents the data mapped by the estimating optimal transformations. Combining the equation(13) and LRR models, the EOTLRR model can be defined as

$$
\min _{\mathbf{Z}}\|\mathbf{Z}\|_{*} \quad \text { s.t. } \varphi(\mathbf{X})=\varphi(\mathbf{X}) \mathbf{Z}
$$

In fact, there is always noise within data. This may result in a bad similarity matrix $\mathbf{Z}$. Therefore, we introduce matrix $\mathbf{E}$ into the method to eliminate the effect of noise, and the problem(14) can be changed to,

$$
\min _{\mathbf{Z}, \mathbf{E}}\|\mathbf{Z}\|_{*}+\lambda\|\mathbf{E}\|_{2,1} \quad \text { s.t. } \varphi(\mathbf{X})=\varphi(\mathbf{X}) \mathbf{Z}+\mathbf{E}
$$


where $\|\cdot\|_{2,1}$ is the $l_{2,1}$-norm and $\lambda$ is the balance coefficient. To optimal solution of EOTLRR, we first introduce an auxiliary matrix $\mathbf{H}$ so that in the method can be separable, and then rewrite the problem(15) as

$$
\min _{\mathbf{Z}, \mathbf{E}}\|\mathbf{Z}\|_{*}+\lambda\|\mathbf{E}\|_{2,1} \quad \text { s.t. } \varphi(\mathbf{X})=\varphi(\mathbf{X}) \mathbf{Z}+\mathbf{E} \quad \mathbf{Z}=\mathbf{H}
$$

\section{3 solving EOTLRR}

The optimization of problem (16) can be solved by using the augmented Lagrange function (J. Yang \& Yuan, 2013).The augmented Lagrange function of the problem (16) can be expressed as follows,

$$
\begin{aligned}
& L\left(\mathbf{Z}, \mathbf{E}, \mathbf{H}, \mathbf{M}_{1}, \mathbf{M}_{2}, \mu\right) \\
= & \|\mathbf{Z}\|_{*}+\lambda\|\mathbf{E}\|_{2,1}+\left\langle\mathbf{M}_{1}, \varphi(\mathbf{X})-\varphi(\mathbf{X}) \mathbf{Z}-\mathbf{E}\right\rangle+\left\langle\mathbf{M}_{2}, \mathbf{Z}-\mathbf{H}\right\rangle \\
+ & \frac{\mu}{2}\left(\|\varphi(\mathbf{X})-\varphi(\mathbf{X}) \mathbf{Z}-\mathbf{E}\|_{F}^{2}+\|\mathbf{Z}-\mathbf{H}\|_{F}^{2}\right)
\end{aligned}
$$

Where $\mathbf{M}_{1}, \mathbf{M}_{2}$ is the Lagrange multiplier, $\mu>0$ is the penalty coefficient, $\|\cdot\|_{F}$ is the Frobenius norm of the matrix, and $\langle\cdot, \cdot\rangle$ refers to the inner product. Problem (17) can be simplified into

$$
\begin{aligned}
& L\left(\mathbf{Z}, \mathbf{E}, \mathbf{H}, \mathbf{M}_{1}, \mathbf{M}_{2}, \mu\right) \\
= & \|\mathbf{H}\|_{*}+\lambda\|\mathbf{E}\|_{2,1}+\frac{\mu}{2}\left(\left\|\varphi(\mathbf{X})-\varphi(\mathbf{X}) \mathbf{Z}-\mathbf{E}+\frac{\mathbf{M}_{1}}{\mu}\right\|_{F}^{2}+\left\|\mathbf{Z}-\mathbf{H}+\frac{\mathbf{M}_{2}}{\mu}\right\|_{F}^{2}\right)
\end{aligned}
$$

Alternating Direction Method (ADM) can be used to alternately solve the optimization of the problem (18). We can fix other variables to alternately update $\mathbf{Z , E} \mathbf{E} \mathbf{H}$, the process is as follows,

$$
\begin{gathered}
\mathbf{Z}_{k+1}=\left(\varphi(\mathbf{X})^{T} \varphi(\mathbf{X})+I\right)^{-1}\left(\varphi(\mathbf{X})^{T} \varphi(\mathbf{X})-\varphi(\mathbf{X})^{T} \mathbf{E}_{k}+\mathbf{J}_{k}+\left(\varphi(\mathbf{X})^{T} \mathbf{M}_{1 k}-\mathbf{M}_{2 k}\right) / \mu_{1 k}\right) \\
\mathbf{E}_{k+1}=\underset{\mathbf{E}}{\arg \min }\|\mathbf{E}\|_{2,1}+\frac{\mu}{2}\left\|\varphi(\mathbf{X})-\varphi(\mathbf{X}) \mathbf{Z}_{k}-\mathbf{E}_{k}+\frac{\mathbf{M}_{1 k}}{\mu}\right\|_{F}^{2} \\
\mathbf{H}_{k+1}=\underset{\mathbf{H}}{\arg \min }\left\|\mathbf{Z}_{k}-\mathbf{H}_{k}+\frac{\mathbf{M}_{2 k}}{\mu}\right\|_{F}^{2}
\end{gathered}
$$

Although the problem (20) and (21) are convex problems, they can get closed-form solution. Problem (21) can be solved by the Singular Value Decomposition (SVD) operator (Drineas, Frieze, Kannan, Vempala, \& Vinay, 2004), and problem (20) can be solved by the following lemma 1.

$\mathbf{1}$ Let $\mathbf{S}$ is a given matrix. If the optimal solution of

is $\mathbf{J}^{*}$, then the $i$-th column of $\mathbf{J}^{*}$ is

$$
\min \alpha\|\mathbf{J}\|_{2,1}+\frac{1}{2}\|\mathbf{J}-\mathbf{S}\|_{F}^{2}
$$

$$
\left[\mathbf{J}^{*}\right]_{:, i}= \begin{cases}\frac{\left\|[\mathbf{S}]_{:, i}\right\|_{2}-\alpha}{\left\|[\mathbf{S}]_{:, i}\right\|_{2}} \mathbf{S}_{:, i}, & \text { if }\left\|[\mathbf{S}]_{:, i}\right\|_{2}>\alpha \\ 0, & \text { otherwise }\end{cases}
$$


The iteration of other parameters is shown in the following formulas,

$$
\begin{gathered}
\mathbf{M}_{1 k+1}=\mathbf{M}_{1 k}+\mu_{1 k}\left(\mathbf{X}-\mathbf{A} \mathbf{Z}_{k+1}-\mathbf{E}_{k+1}\right) \\
\mathbf{M}_{2 k+1}=\mathbf{M}_{2 k}+\mu_{1 k}\left(\mathbf{Z}_{k+1}-\mathbf{H}_{k+1}\right) \\
\mu_{1 k+1}=\min \left(\rho \mu_{1 k}, \mu_{2}\right)
\end{gathered}
$$

In the EOTLRR algorithm, firstly, we use the estimating optimal transformations for nonlinear data transformation to enhance the correlation between samples belonging to the same class. The specific procedure of realizing the nonlinear transformation is shown in algorithm 2. After that, the similarity matrix is obtained using the transformed data and it can be found in Algorithm3. After obtaining the similarity matrix $\mathbf{Z}^{*}$ through the EOTLRR model, then the affinity matrix through $\mathbf{W}=\left(\left|\mathbf{Z}^{*}\right|+\left|\mathbf{Z}^{*}\right|^{\prime}\right) / 2$ is constructed. Finally, we use unsupervised classification methods $\mathrm{N}$-cut method to obtain the final clustering results.

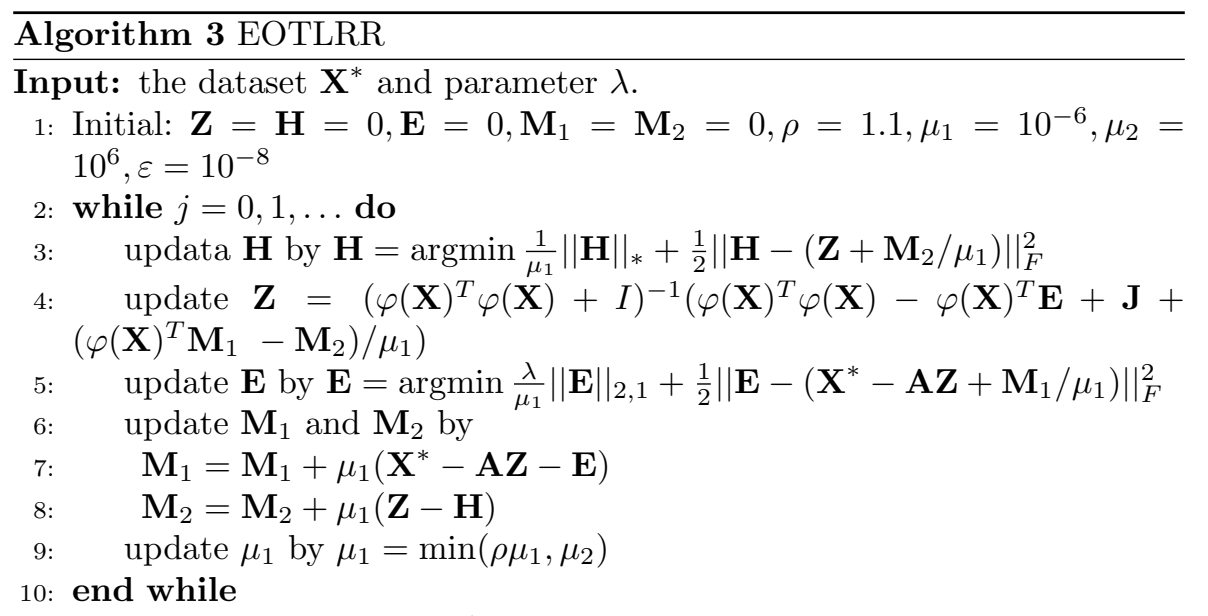

Output: Similarity matrix $\mathbf{Z}^{*}$.

\section{Experiments}

In this section, to verify the effectiveness of our proposed algorithm on the clustering problem, the proposed method has been tested on five real datasets and an artificial dataset. All the experiments are implemented on MATLAB 2018b, a PC with Intel (R) Xeon (R) Platinum 8269 cy CPU, 2.5 GHz, RAM $16.00 \mathrm{~Gb}$ and windows 10 operating system.

In the experiment, the datasets we tested include Swiss, Extended Yale B, ORL, COIL20, MNIST, and USPS. Among them, the Swiss Roll is a synthetic dataset. The Extended Yale B and ORL are two facial image datasets. The COIL20 contains different objects. The MNIST and USPS are two handwritten datasets composed of numbers. 


\subsection{Image clustering}

On each dataset, we compare the proposed method with the following methods:

- Sparse Subspace Clustering (SSC) (Elhamifar \& Vidal, 2012)

- Low-Rank Representation (LRR) (G. Liu et al., 2010)

- Dual Graph Regularized LRR (DGLRR) (Yin, Gao, Lin, Shi, \& Guo, 2015)

- Non-Convex LRR (NLRR) (Jie \& Ping, 2016)

- Robust Kernel LRR (RKLRR) (Xiao et al., 2016)

- Hierarchical Weighted LRR (HWLRR) (Fu, Zhao, Chang, \& Wang, 2021)

- Adaptive Low-Rank Representation (ALRR) (Chen, Mao, Wang, \& Zhang, 2021)

All the codes involved in the comparison algorithm are from their respective authors. To make a fair comparison, the comparison algorithm achieves the best result by manually adjusting the parameters according to the parameter settings provided in the corresponding paper. LRR and SSC are two classic model-based representations. DGLRR take the geometric structure of data manifold and feature manifold into consideration and obtains better clustering results. NLRR decomposes the regularization of the kernel norm, which improves the clustering performance. After mapping the original samples from nonlinear space to multiple linear low dimensional subspaces using the kernel technology through RKLRR, we can obtain a better similarity matrix. HWLRR defines a matrix through the affinity propagation to find samples that may belong to the same subspace. By using an adaptive dictionary learning strategy that employs orthonormality constraint and integrated into the LRR model, ALRR is gained. Each comparision method obtains a representation matrix through corresponding algorithm. Based on this, we can adopt N-cut to get the sample label. Since most clustering evaluation criteria are determined by the accuracy, so we use it as the reference. All algorithms are performed 10 times, and then the average clustering results are analyzed and compared. The clustering result of different methods on the data set is shown in Table $1-6$.

\subsection{Datasets}

\subsubsection{Experiments on the Synthetic Dataset}

We test the performance of different algorithms on clean noisy synthetic data by adding different degrees of noise to Swiss data. The data generation process is as follows: a $2 \times 2$ covariance matrix is generated by the Gaussian mixture model, and it is comprised of 2 dimensional point set for 4 categories. 400 linear separable data are collected from each point set to form a $1600 \times 2$ matrix, and then $2-\mathrm{D}$ data is mapped to 3 -D space by smooth mapping to form Swiss. What we get is a clean data matrix that is nonlinear separable. Fig 2 shows the visualization of the dataset. Next, we further destroy the data by adding different degrees of normal distribution random numbers to all clean data. Table 1 shows the average accuracy of the five methods relative to the 

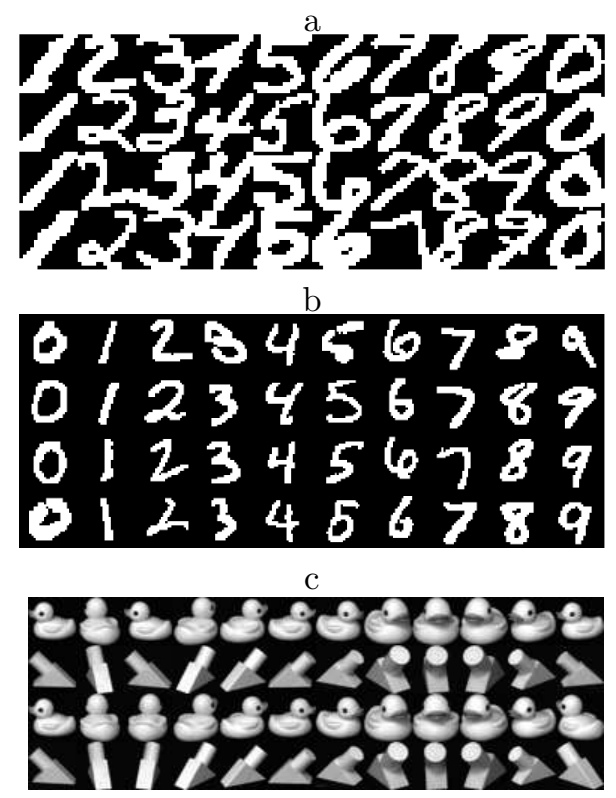

Fig. 1 Example images of multiple individuals from the USPS dataset, MNIST dataset and the COIL20 dataset. (a)partial images of the USPS; (a)partial images of the MNIST; (b)partial images of the COIL20.

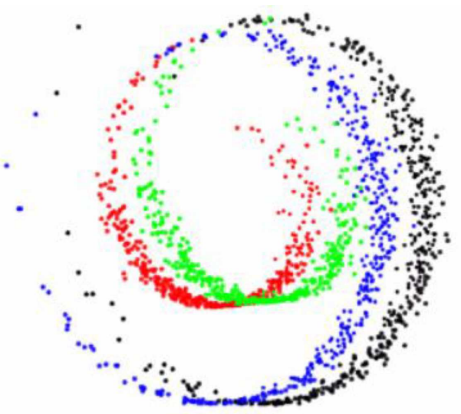

Fig. 2 Three-dimensional Swiss roll data generated by random numbers, with different colors indicating different categories.

corruption rate. It can be seen from Table 1 that the proposed method has the best performance and can better handle non-linear and noisy data.

\subsubsection{Experiments on the Extended Yale B}

The extended Yale B dataset (http://www.cad.zju.edu.cn/home/dengcai/ Data/FaceData.html) is a standard dataset for the facial clustering task. It includes 2414 face images of 38 people. We downsampled the original image of $192 \times 168$ pixels to $48 \times 42$ pixels to reduce the computation time. Therefore, each face image can be vectorized into a 2016 dimensional vector as a sample. 
Table 1 Clustering performance index (average) of different methods in Synthetic dataset. Bold numbers indicate the best results. $P$ denotes the percentage of corruptions

\begin{tabular}{lllllll}
\hline$P(\%)$ & 0 & 20 & 40 & 60 & 80 & 100 \\
\hline SSC & 0.4732 & 0.4881 & 0.4838 & 0.4606 & 0.4800 & 0.4912 \\
LRR & 0.3975 & 0.3962 & 0.3919 & 0.3887 & 0.3869 & 0.3825 \\
DGLRR & 0.4569 & 0.4531 & 0.4522 & 0.4511 & 0.3925 & 0.3875 \\
NLRR & 0.5269 & 0.5194 & 0.5144 & 0.4925 & 0.4912 & 0.4719 \\
RKLRR & 0.5269 & 0.5794 & 0.5144 & 0.4925 & 0.4912 & 0.4719 \\
HWLRR & 0.5094 & 0.4985 & 0.4933 & 0.4921 & 0.3912 & 0.3879 \\
ALRR & 0.4867 & 0.4294 & 0.4313 & 0.4463 & 0.4569 & 0.4319 \\
EOTLRR & $\mathbf{0 . 9 5 9 4}$ & $\mathbf{0 . 9 5 8 1}$ & $\mathbf{0 . 9 5 6 9}$ & $\mathbf{0 . 9 5 6 3}$ & $\mathbf{0 . 9 5 4 4}$ & $\mathbf{0 . 9 5 1 9}$ \\
\hline
\end{tabular}

Table 2 Clustering performance index (average) of different methods in ExtYale B dataset. Bold numbers indicate the best results. $K$ denotes the number of category of dataset.

\begin{tabular}{llllll}
\hline$K$ & 2 & 4 & 6 & 8 & 10 \\
\hline SSC & 0.9766 & 0.9896 & 0.9453 & 0.8535 & 0.7766 \\
LRR & 0.9766 & 0.9609 & 0.9688 & 0.9082 & 0.8797 \\
DGLRR & 0.9609 & 0.8805 & 0.7464 & 0.6836 & 0.5328 \\
NLRR & 0.6688 & 0.6328 & 0.6250 & 0.6140 & 0.5766 \\
RKLRR & 0.6688 & 0.6406 & 0.6354 & 0.6328 & 0.5109 \\
HWLRR & 0.9766 & 0.9733 & 0.9625 & 0.9640 & 0.7333 \\
ALRR & 0.9922 & $\mathbf{0 . 9 9 2 2}$ & $\mathbf{0 . 9 8 9 6}$ & $\mathbf{0 . 9 7 8 5}$ & $\mathbf{0 . 9 7 5 0}$ \\
EOTLRR & $\mathbf{1 . 0 0 0 0}$ & $\mathbf{0 . 9 9 2 2}$ & 0.9714 & 0.9629 & 0.9609 \\
\hline
\end{tabular}

Table 3 Clustering performance index (average) of different methods in ORL dataset. Bold numbers indicate the best results. $K$ denotes the number of category of dataset.

\begin{tabular}{lllllllll}
\hline$K$ & 5 & 10 & 15 & 20 & 25 & 30 & 35 & 40 \\
\hline SSC & 0.8400 & 0.7200 & 0.7133 & 0.7700 & 0.7800 & 0.7560 & 0.7343 & 0.7175 \\
LRR & 0.4400 & 0.3000 & 0.2667 & 0.2550 & 0.2200 & 0.2200 & 0.1943 & 0.1875 \\
DGLRR & 0.7800 & 0.7200 & 0.6933 & 0.6950 & 0.6400 & 0.6633 & 0.6314 & 0.6175 \\
NLRR & 0.8600 & 0.7700 & 0.7733 & 0.7950 & 0.7560 & 0.7900 & 0.7771 & 0.7175 \\
RKLRR & 0.7800 & 0.7700 & 0.7667 & 0.7600 & 0.7440 & 0.7567 & 0.7571 & 0.7225 \\
HWLRR & 0.4600 & 0.4200 & 0.3000 & 0.1950 & 0.2080 & 0.1767 & 0.2514 & 0.2200 \\
ALRR & 0.9200 & 0.8300 & 0.8533 & 0.7900 & 0.7680 & 0.7667 & 0.7914 & 0.7625 \\
EOTLRR & $\mathbf{1 . 0 0 0 0}$ & $\mathbf{0 . 9 9 4 0}$ & $\mathbf{0 . 9 9 3 3}$ & $\mathbf{0 . 9 9 0 0}$ & $\mathbf{0 . 9 7 2 0}$ & $\mathbf{0 . 9 6 9 3}$ & $\mathbf{0 . 9 4 9 7}$ & $\mathbf{0 . 9 4 5 3}$ \\
\hline
\end{tabular}

Table 4 Clustering performance index (average) of different methods in COIL20 dataset. Bold numbers indicate the best results. $K$ denotes the number of category of dataset.

\begin{tabular}{lllllllll}
\hline$K$ & 2 & 4 & 6 & 8 & 10 & 12 & 16 & 20 \\
\hline SSC & 0.9792 & 0.8715 & 0.7500 & 0.7326 & 0.2920 & 0.7188 & 0.6944 & 0.6333 \\
LRR & 0.9688 & 0.9297 & 0.8218 & 0.7934 & 0.7694 & 0.7176 & 0.6667 & 0.6292 \\
DGLRR & 0.9514 & 0.8542 & 0.7986 & 0.6319 & 0.7736 & 0.6887 & 0.7595 & 0.7111 \\
NLRR & 0.9635 & 0.8333 & 0.6157 & 0.6285 & 0.6097 & 0.6412 & 0.6389 & 0.6528 \\
RKLRR & 0.9931 & 0.8056 & 0.6296 & 0.6042 & 0.6236 & 0.6505 & 0.7109 & 0.6854 \\
HWLRR & 0.9722 & 0.9688 & 0.9297 & 0.8142 & 0.7903 & 0.8032 & 0.6345 & 0.7771 \\
ALRR & 0.9200 & 0.8300 & 0.8600 & 0.7700 & 0.7680 & 0.7633 & 0.8029 & 0.7253 \\
EOTLRR & $\mathbf{0 . 9 9 3 1}$ & $\mathbf{0 . 9 8 6 1}$ & $\mathbf{0 . 9 3 0 6}$ & $\mathbf{0 . 9 3 4 0}$ & $\mathbf{0 . 9 5 4 2}$ & $\mathbf{0 . 9 4 2 1}$ & $\mathbf{0 . 9 2 9 7}$ & $\mathbf{0 . 9 0 2 1}$ \\
\hline
\end{tabular}


Table 5 Clustering performance index (average) of different methods in MNIST dataset. Bold numbers indicate the best results. \# Tr denotes the number of selected samples of each subject.

\begin{tabular}{llllllll}
\hline$\#$ Tr & 30 & 50 & 100 & 150 & 200 & 250 & 300 \\
\hline SSC & 0.4933 & 0.4200 & 0.4060 & 0.4287 & 0.4390 & 0.4120 & 0.4450 \\
LRR & 0.6000 & 0.6640 & 0.5720 & 0.5430 & 0.5650 & 0.5420 & 0.5500 \\
DGLRR & 0.4500 & 0.4860 & 0.4580 & 0.4467 & 0.4785 & 0.4672 & 0.4513 \\
NLRR & 0.6133 & 0.5740 & 0.5620 & 0.5213 & 0.6050 & 0.5132 & 0.5563 \\
RKLRR & 0.6333 & 0.6240 & 0.5950 & 0.6033 & 0.5845 & 0.6252 & 0.6170 \\
HWLRR & 0.5800 & 0.5340 & 0.5340 & 0.5150 & 0.5213 & 0.5368 & 0.5547 \\
ALRR & 0.7700 & 0.7140 & 0.7980 & 0.7453 & 0.8410 & 0.7952 & 0.7913 \\
EOTLRR & $\mathbf{0 . 8 9 3 3}$ & $\mathbf{0 . 9 3 6 0}$ & $\mathbf{0 . 9 4 2 0}$ & $\mathbf{0 . 9 5 8 7}$ & $\mathbf{0 . 9 7 5 5}$ & $\mathbf{0 . 9 6 7 6}$ & $\mathbf{0 . 9 8 7 5}$ \\
\hline
\end{tabular}

Table 6 Clustering performance index (average) of different methods in USPS dataset. Bold numbers indicate the best results. \# Tr denotes the number of selected samples of each subject.

\begin{tabular}{lllllll}
\hline$\#$ Tr & 50 & 100 & 200 & 300 & 500 & 800 \\
\hline SSC & 0.4980 & 0.5510 & 0.6030 & 0.4463 & 0.4043 & 0.3622 \\
LRR & 0.5500 & 0.8080 & 0.7220 & 0.8960 & 0.5030 & 0.4865 \\
DGLRR & 0.3430 & 0.4810 & 0.4860 & 0.4397 & 0.4434 & 0.4126 \\
NLRR & 0.5500 & 0.5160 & 0.5080 & 0.5017 & 0.5030 & 0.4865 \\
RKLRR & 0.5260 & 0.5990 & 0.6225 & 0.5800 & 0.5908 & 0.5340 \\
HWLRR & 0.4540 & 0.6480 & 0.6700 & 0.6347 & 0.6288 & 0.3965 \\
ALRR & 0.5720 & 0.6790 & 0.6820 & 0.6903 & 0.6832 & 0.7263 \\
EOTLRR & $\mathbf{0 . 8 1 0 0}$ & $\mathbf{0 . 8 1 2 0}$ & $\mathbf{0 . 8 2 0 0}$ & $\mathbf{0 . 8 8 2 7}$ & $\mathbf{0 . 8 9 6 0}$ & $\mathbf{0 . 9 5 5 9}$ \\
\hline
\end{tabular}

In the experiments, we randomly select $2,4,6,8,10$ classes from 38 kinds of face images for experiments. For each selected cluster class $\mathbf{K}$, the experimental results are revealed in Table 2, it can be seen that our method has a higher recognition rate in most cases.

\subsubsection{Experiments on the ORL Dataset}

ORL face dataset (Samaria \& Harter, 1994) (http://www.cl.cam.ac.uk/ research/dtg/attarchive/facedatabase.html) is composed of 400 face images, each category of 10 face images, 40 people included. According to the processing of the ORL face dataset in the paper (Cai, He, Hu, Han, \& Huang, 2007), the ORL dataset is downsampled, and the original image is sampled from $112 \times 92$ to $32 \times 32$. For each category, these images are gained under different expressions and conditions like open/close eyes, smile/no smile. Experiments randomly select $5,10,15,20,25,30,35,40$ classes from whole 40 kinds of face images for experiments. Clustering on this dataset is a challenging problem because of many different expressions and details. The clustering results of different methods are shown in Table 3. It can be seen that our proposed method performs best. 


\subsubsection{Experiments on the COIL20 Dataset}

COIL20 is an object image dataset (http://www.cs.columbia.edu/CAVE/ software/softlib/coil-20.php), which contains 1440 images of 20 objects, including duck, car model, and others. Each object is photographed every five degrees horizontally, and 72 images are obtained for each subject. For improving the computational efficiency, we adjust the $128 \times 128$ pixels of the original image to $32 \times 32$ pixels of the gray image and then convert each image into 1024 dimensional feature vectors. Examples of partial images of two objects selected from the COIL20 dataset are shown in Fig 1 . We randomly select $2,4,6,8,10,12,16,20$ classes from all kinds of object images for experiments. As demonstrated in Table 4, the proposed method presents a higher clustering accuracy.

\subsubsection{Experiments on the MNIST Dataset}

MNIST (Lecun, Bottou, Bengio, \& Haffner, 1998) is a handwritten digital dataset (http://yann.lecun.com/exdb/mnist/) that contains images of 10 handwritten digits from 0 to 9 . This dataset contains 60000 training images and 10000 test images. Each image is $28 \times 28$ pixels in size. We establish a 784 dimensional vector for all pixels of every image. Some images of each digit from the MNIST are shown in the first row in Fig 1 . We randomly select $30,50,100,150,200,300$ for each number as sample sets for clustering. The experimental results are displayed in Table 5 . It can be seen from the data in the table that EOTLRR can get the best performance.

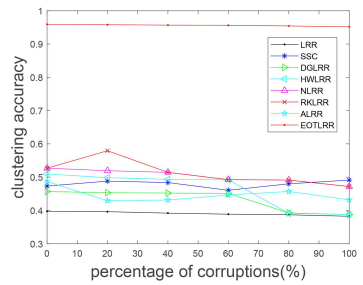

(a) Synthetic Dataset

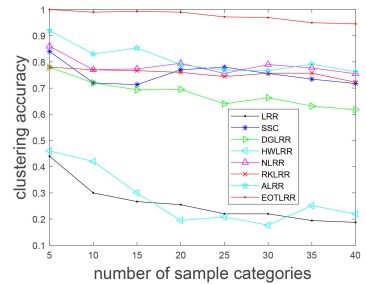

(d) ORL

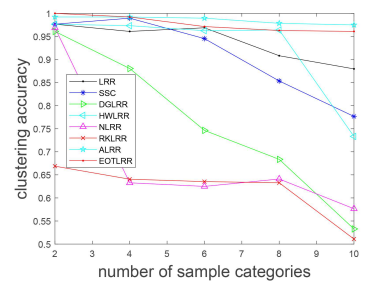

(b) Extended Yale B

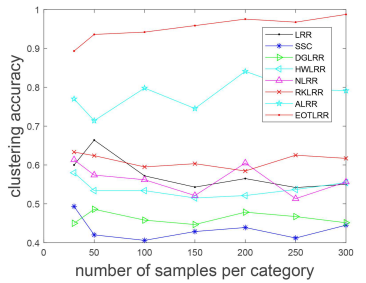

(e) MNIST

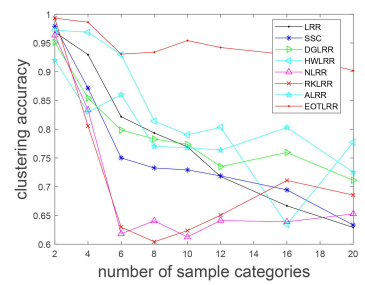

(c) COIL20

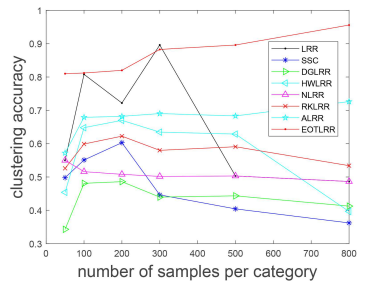

(f) USPS

Fig. 3 Line graphs of experimental results of different methods on synthetic dataset and five real datasets. 


\subsubsection{Experiments on the USPS Dataset}

USPS (Hull, 1994) is a handwritten dataset containing 9828 samples. It consist of 10 categories of samples, which are numbers $0-9$, a proportion images for each number are shown in Fig 1 . Each sample is a $16 \times 16$ normalized gray image, so each sample image can be represented as a 256 dimensional vector. Given the data sample is too many, we randomly select $50,100,200,300,500,800$ images for each number as the sample sets to experiment. It can be seen from Table 6 that our proposed method has better performance than the comparing methods.

\subsection{Experimental results analysis}

Fig 3 shows the clustering results of the proposed method and comparison methods on a synthetic dataset and the five benchmark datasets. It can be seen from the Fig 3 that the proposed method obtains the best clustering performance in most cases, and the proposed method has better stability with the increase of samples or categories, which proves that the nonlinear mapping of the original data can better measure the similarity among samples.

The results on the Swiss show that the proposed EOTLRR method is more robustness for data with nonlinear structure and Gaussian noise. Compared with experimental results of other methods on the Swiss, it can be seen that the proposed method can better deal with nonlinear structure and Gaussian noise data. Among them, the purpose of the methods RKLRR and DGLRR are to handle nonlinear structure data. Nevertheless, the experimental results on Swiss show that their ability to handle nonlinear data is not ideal, it may be because the selected kernel function and regular graph are not suitable for this dataset.

In addition, the experimental results on real datasets show that the proposed method is the best in most cases. Because the noise and nonlinear structure in the real dataset is uncertain, which is enough to demonstrate that EOTLRR is an effective method. We observed that ALRR achieved the more result in the ExtYaleB dataset when the number of samples increases. This indicates that the adaptive dictionary learning strategy of ALRR plays an important role in its data matrix representation when the number of data samples is being increased. But the clustering results on the other datasets show that EOTLRR exhibits a stronger data clustering ability than the competing algorithms as the number of clusters grows.

We also noticed that the performance of the proposed method has been significantly improved when the number of samples in each category is small on the other datasets. For instance there are only 10 samples per people in the ORL, it can be seen in Fig 3(d) that the accuracy of our proposed method is the highest. Besides, in the USPS and MNIST handwritten digit datasets, the proposed method still has the best-fit result when the number of samples used is small, and the accuracy rate will increase with the samples of increasing. Furthermore, when the number of samples is sufficient, as the categories 
increases, the proposed method can still get a better result. In a word, the EOTLRR is suitable for data with nonlinear spatial structure and noisy data. The main reason is that EOTLRR can map nonlinear structure data to linear structure data, which effectively improves the correlation of samples belonging to same class, and can remove the influence of some outliers and noise, thereby constructing a more discriminative affinity matrix.

\section{Conclusions}

In this paper, a new nonlinear low-rank representation method is proposed, using the estimating optimal transformations to increase the correlation between samples that may belong to the same category. In EOTLRR, the data is first mapped from a non-linear structure to a linear structure by estimating the optimal transformation function, and the ACE method is used to optimize this nonlinear transformation function. Using a nonlinear transformation function on the original data can enhance the correlation between samples that may belong to the same category in the proposed method. Obtained by the method proposed by affinity matrix and well preserved both the global structure data, but also better capture the local structure of the entire data. Experimental results on multiple datasets show the effectiveness of this method.

\section{References}

Breiman, L., \& Friedman, J.H. (1985a). Estimating optimal transformations for multiple regression and correlation. Journal of the American statistical Association, 80(391), 580-598.

Breiman, L., \& Friedman, J.H. (1985b). Estimating optimal transformations for multiple regression and correlation. Journal of the American Statistical Association, 80(391), 614-619.

Cai, D., He, X., Hu, Y., Han, J., Huang, T. (2007). Learning a spatially smooth subspace for face recognition. 2007 ieee conference on computer vision and pattern recognition (pp. 1-7).

Chen, J., Mao, H., Wang, Z., Zhang, X. (2021). Low-rank representation with adaptive dictionary learning for subspace clustering. Knowledge-Based Systems, 223, 107053.

Chen, J., Mao, H., Zhang, H., Yi, Z. (2018). Symmetric low-rank preserving projections for subspace learning. Neurocomputing, 315, 381-393. 
Drineas, P., Frieze, A., Kannan, R., Vempala, S., Vinay, V. (2004). Clustering large graphs via the singular value decomposition. Machine Learning, $56(1 / 3), 9-33$.

Du, H., Ma, L., Li, G., Wang, S. (2020). Low-rank graph preserving discriminative dictionary learning for image recognition. Knowledge-Based Systems, 187, 104823.

Elhamifar, E., \& Vidal, R. (2012). Sparse subspace clustering: Algorithm, theory, and applications. IEEE Transactions on Pattern Analysis 86 Machine Intelligence, 35(11), 2765-2781.

Elhamifar, E., \& Vidal, R. (2013). Sparse subspace clustering: Algorithm, theory, and applications. IEEE Transactions on Pattern Analysis \&5 Machine Intelligence, 35(11), 2765-2781.

Feizi, S., \& Tse, D. (2017). Maximally correlated principal component analysis. arXiv:1702.05471v2.

Feng, L., Meng, X., Wang, H. (2020). Multi-view locality low-rank embedding for dimension reduction. Knowledge-Based Systems, 191, 105172.

Fu, Z., Zhao, Y., Chang, D., Wang, Y. (2021). A hierarchical weighted low-rank representation for image clustering and classification. Pattern Recognition, 112(7).

Hull, J.J. (1994). A database for handwritten text recognition research. IEEE Transactions on Pattern Analysis and Machine Intelligence, 16(5), 550554 .

Jie, S., \& Ping, L. (2016). Learning structured low-rank representation via matrix factorization. International conference on artificial intelligence E statistics.

Lauer, F., \& Schnorr, C. (2009). Spectral clustering of linear subspaces for motion segmentation. Ieee international conference on computer vision. 
Nonlinear low rank representation by estimating optimal transformations for image clusteri

Lecun, Y., Bottou, L., Bengio, Y., Haffner, P. (1998). Gradient-based learning applied to document recognition. Proceedings of the IEEE, 11, 22782324 .

Li, X., Cui, G., Dong, Y. (2017). Graph regularized non-negative lowrank matrix factorization for image clustering. IEEE Transactions on Cybernetics, 47(11), 3840-3853.

Liu, G., Lin, Z., Yan, S., Sun, J., Yu, Y., Ma, Y. (2010). Robust recovery of subspace structures by low-rank representation. Pattern Analysis and Machine Intelligence, IEEE Transactions on, 35(1), 171-184.

Liu, G., Lin, Z., Yan, S., Sun, J., Yu, Y., Ma, Y. (2013). Robust recovery of subspace structures by low-rank representation. IEEE Transactions on Pattern Analysis and Machine Intelligence, 35(1), 171-184.

Liu, G., \& Yan, S. (2011). Latent low-rank representation for subspace segmentation and feature extraction. 2011 international conference on computer vision (pp. 1615-1622).

Liu, R., Lin, Z., la Torre, F.D., Su, Z. (2012). Fixed-rank representation for unsupervised visual learning. 2012 ieee conference on computer vision and pattern recognition (pp. 198-605).

Michael, E.T., \& Christopher, M.B. (1999). Mixtures of probabilistic principal component analyzers. Neural Computation, 11(2), 443-482.

Peng, X., Feng, J., Xiao, S., Yau, W., Zhou, J.T., Yang, S. (2018). Structured autoencoders for subspace clustering. IEEE Transactions on Image Processing, 27(10), 5076-5086.

Peng, X., Zhu, H., Feng, J., Shen, C., Zhang, H., Zhou, J.T. (2020). Deep clustering with sample-assignment invariance prior. IEEE Transactions on Neural Networks and Learning Systems, 31(11), 4857-4868.

Samaria, F.S., \& Harter, A.C. (1994). Parameterisation of a stochastic model for human face identification. Proceedings of 1994 ieee workshop on applications of computer vision (pp. 138-142). 
Shamir, O., \& Tishby, N. (2010, SEP). Stability and model selection in k-means clustering [Article]. Machine Learning, 80(2-3, SI), 213-243.

Shi, J., \& J.Malik. (2000). Normalized cuts and image segmentation. IEEE Transactions on Pattern Analysis $\&$ Machine Intelligence, 22(8), 888905.

Vidal, R., Ma, Y., Sastry, S. (2005). Generalized principal component analysis (gpca). IEEE Transactions on Pattern Analysis and Machine Intelligence, 27(12), 1945-1959.

Wang, J., Saligrama, V., A., D. (2011). Structural similarity and distance in learning. 2011 49th annual allerton conference on communication, control, and computing (allerton) (pp. 744-751).

Xiao, S., Tan, M., Xu, D., Dong, Z.Y. (2016). Robust kernel low-rank representation. IEEE Transactions on Neural Networks and Learning Systems, 27(11), 2268-2281.

Xue, Z., Du, J., Du, D., Li, G., Huang, Q., Lyu, S. (2019). Deep constrained low-rank subspace learning for multi-view semi-supervised classification. IEEE Signal Processing Letters, 26(8), 1177-1181.

Yang, A.Y., Rao, S.R., Yi, M. (2006). Robust statistical estimation and segmentation of multiple subspaces. Conference on computer vision 85 pattern recognition workshop.

Yang, J., \& Yuan, X. (2013). Linearized augmented lagrangian and alternating direction methods for nuclear norm minimization. Math. Comp. 82, 281, 301-329.

Yin, M., Gao, J., Lin, Z. (2016). Laplacian regularized low-rank representation and its applications. IEEE Transactions on Pattern Analysis and Machine Intelligence, 38(3), 504-517.

Yin, M., Gao, J., Lin, Z., Shi, Q., Guo, Y. (2015). Dual graph regularized latent low-rank representation for subspace clustering. IEEE Transactions on Image Processing, 24(12), 4918-4933. 
Nonlinear low rank representation by estimating optimal transformations for image clusteri

Yu, J., Tan, M., Zhang, H., Tao, D., Rui, Y. (2019). Hierarchical deep click feature prediction for fine-grained image recognition. IEEE Transactions on Pattern Analysis and Machine Intelligence, 1-1.

Yu, J., Tao, D., Wang, M., Rui, Y. (2015). Learning to rank using user clicks and visual features for image retrieval. IEEE Transactions on Cybernetics, 45(4), 767-779.

Zhang, H., Lin, Z., Zhang, C., Gao, J. (2014). Robust latent low rank representation for subspace clustering. Neurocomputing, 145, 369-373.

Zhang, J., Li, X., Jing, P., Liu, J., Su, Y. (2018). Low-rank regularized heterogeneous tensor decomposition for subspace clustering. IEEE Signal Processing Letters, 25(3), 333-337.

Zhong, G., \& Pun, C. (2020). Subspace clustering by simultaneously feature selection and similarity learning. Knowledge-Based Systems, 193, 105512 .

Zhu, W., \& Peng, B. (2020). Sparse and low-rank regularized deep subspace clustering. Knowledge-Based Systems, 204, 106199.

\section{Statements and Declarations}

\section{Funding}

The work is supported by the Natural Science Foundation of China under Grant No. 61906175, and the Key Technologies R\&D Program of Henan Province under Grant No. 212102210409. Support the research information supported by the first author Haoran Chen and Yong Cui.

\section{Competing Interests}

The authors have no relevant financial or non-financial interests to disclose.

\section{Author Contributions}

Hongwei Tao and Zuhe $\mathrm{Li}$ analyzed and interpreted the experiment data regarding in the five datasets. Haoran Chen and Xu Chen performed the build method and optimal, and was a major contributor in writing the manuscript. 
Yong Cui and Hanming Guo adjusted the grammar and sentence structure of the paper. All authors read and approved the final manuscript.

\section{Data Availability}

The dataset generated during the current study are available from the corresponding author on reasonable request.

\section{Ethics approval}

Not Applicable

\section{Code availability}

The code are available from the corresponding author on reasonable request.

\section{Consent for publication}

The Author agrees to publication in the Journal indicated below and also to publication of the article in English by Springer in Springer's corresponding English-language journal. 